\title{
MicroRNA-758 inhibits cervical cancer cell proliferation and metastasis by targeting HMGB3 through the WNT/ $\beta$-catenin signaling pathway
}

\author{
TAO SONG ${ }^{1}$, XINGHUA HOU ${ }^{2}$ and BING LIN ${ }^{1}$ \\ Departments of ${ }^{1}$ Gynecology and ${ }^{2}$ Women Health Care, Weifang Maternity and \\ Child Care Hospital, Weifang, Shandong 261042, P.R. China \\ Received October 14, 2018; Accepted February 19, 2019
}

DOI: $10.3892 / 01.2019 .10470$

\begin{abstract}
Cervical cancer (CC) remains a highly prevalent cancer and cause of mortality amongst women worldwide. miR-758 has been demonstrated to be associated with tumorigenesis by controlling the expression of oncogenic or tumor suppressor genes. However, the function and mechanisms of miR-758 in CC have not been well illustrated. The present study aimed to dissect the effect of miR-758 on the proliferation, migration and invasion of $\mathrm{CC}$ cells and determine the potential underlying molecular mechanism of these effects. qPCR results revealed that the expression of miR-758 was significantly decreased in CC tissues and cell lines compared with that in normal tissues and normal cells. Results of CCK-8, colony formation and Transwell assays revealed that miR-758 overexpression markedly decreased cell viability, proliferation, invasion and migration. However, miR-758 inhibitors significantly increased viability, proliferation, invasion and migration. In the mechanism study, we demonstrated that high mobility group box 3 (HMGB3) was a direct target of miR-758, and HMGB3 overexpression rescued the viability, proliferation, invasion and migration of HeLa cells reduced by an miR-758 mimic. It was demonstrated that HMGB3 regulated the WNT/ $\beta$-catenin signaling pathway under miR-758 regulation. In summary, these observations suggested that miR-758 is a tumor suppressor gene that can inhibit the metastatic phenotype of CC cells by negatively regulating HMGB3, which may present a path to novel therapeutic stratagems for CC therapy.
\end{abstract}

Correspondence to: Dr Xinghua Hou, Department of Women Health Care, Weifang Maternity and Child Care Hospital, 407 Qingnian Road, Weifang, Shandong 261042, P.R. China E-mail:wffyhxh@163.com

Key words: microRNA-758, cervical cancer, proliferation, migration and invasion, HMGB3, WNT/ $\beta$-catenin signaling pathway

\section{Introduction}

Cervical cancer (CC) is the third most prevalent gynecological malignancy and the second leading cause of cancer-associated mortality amongst females worldwide with an estimated 530,000 mortalities per year $(1,2)$. Despite developments in radiotherapy, chemotherapy and surgery for the treatment of CC, the 5-year survival rate for patients with CC is still low $(2,3)$. Therefore, the underlying molecular mechanisms of the initiation and progression of CC must be explored, and potential therapeutic strategies should be identified.

MicroRNAs (miRNAs) are small non-coding RNA sequences with 18-21 nucleotides that modulate translational efficiency or stability by targeting the 3'-untranslated regions (3'-UTRs) of mRNAs (3). miRNAs serve a key role in the progression and oncogenesis of a variety of cancers, including CC $(4,5)$. In CC, numerous miRNAs are involved in cancer initiation, promotion and progression (4). The aberrant expression of miR-758 is closely related to glioma, hepatocellular carcinoma and non-small lung cancer (6-8). miR-758 may serve as a tumor suppressor and inhibit CC metastasis (9). However, the biological function and molecular mechanism of miR-758 in CC have not been well illustrated.

In the present study, miR-758 was markedly decreased in primary CC tumor tissues and cell lines. In vitro analysis demonstrated that miR-758 inhibited cell proliferation, migration and invasion in CC cells. High mobility group box 3 (HMGB3) was identified as a direct target of miR-758, and it was involved in miR-758-regulated cell progression. The present study revealed that miR-758 could negatively regulate the WNT/ $\beta$-catenin signaling pathway. The present study was the first to provide novel clues regarding the role of miR-758 as a tumor suppressor gene by regulating HMGB3 in CC.

\section{Materials and methods}

Tissue collection. Human cervical cancer tissues and paired normal cervical tissues were collected from 20 patients (stage I,5 patients; stage II,10 patients; stage III,5 patients) with cervical cancer who were admitted to the Department of Gynecology, Weifang Maternity and Child Care Hospital between January 2017 to December 2017, and written informed 
consent was obtained from all patients. Patients age range was between 42 and 71 years; mean age $52.23 \pm 20.13$ years. Seventeen patients were diagnosed with squamous cell carcinoma; while the other 3 patients were adenocarcinoma. No patients previously received anticancer treatment, including chemotherapy and radiotherapy. This study involving human samples was approved by the Medical Ethics Committee of Weifang Maternity and Child Care Hospital (Weifang, China).

Cell culture. CC cell lines (HeLa, C33A, CaSki and SiHa) and a normal cervical normal cervical cell line Ect1/E6E7 were obtained from the American Type Culture Collection (Manassas, VA, USA). 293T cells were purchased from the Cell Biology of the Chinese Academy of Sciences (Shanghai, China). Cell identity was confirmed by STR analysis. All cell lines were cultured in Dulbecco's modified Eagle's medium (DMEM) (HyClone; GE Healthcare Life Sciences, Logan, UT, USA) with $10 \%$ fetal bovine serum (FBS; HyClone; GE Healthcare Life Sciences) in a humidified atmosphere containing $5 \% \mathrm{CO}_{2}$ at $37^{\circ} \mathrm{C}$.

Cell transfection. The miR-758 mimic (5'-UUUGUGACC UGGUCCACUAACC-3'), corresponding controls (miR-NC) (5'-TTCTCCGAACGTGTCACGT-3'), miR-758 inhibitor (5'-GGUUAGUGGACCAGGUCACAAA-3'), inhibitor control (anti-NC) (5'-UUCUCCGAACGUGUCACGUTT-3') were synthesized by Guangzhou RiboBio Co., Ltd. (Guangzhou, China). A HMGB3 overexpression plasmid and control vector were obtained from Santa Cruz Biotechnology, Inc. (Santa Cruz, CA, USA). During cell transfection, the cells were seeded into 6-well plates, and then cultured until 50-70\% confluency was reached in 1 day. Transient transfection was performed using Lipofectamine 2000 Reagent (Invitrogen; Thermo Fisher Scientific, Inc., Waltham, MA, USA) referring to the manufacturer's protocols.

Cell viability assay. Cell viability was assessed with a Cell Counting Kit-8 (CCK-8) assay (Beyotime Institute of Biotechnology, Haimen, China). Briefly, HeLa and C33A cells were plated onto 96-well plates at a density of 3,000 cells/well. Following culture for the indicated time-points $(0,24,48$ and $72 \mathrm{~h}), 10 \mu \mathrm{l} \mathrm{CCK}-8$ solution was added into each well and incubated at $37^{\circ} \mathrm{C}$. After $3 \mathrm{~h}$, the absorbance of each well was measured using a Multiskan MK3 spectrophotometer (Thermo Fisher Scientific, Inc.) at a wavelength of $450 \mathrm{~nm}$.

Colony formation assay. Cell proliferation was analyzed by colony formation assay. Cells (500) were seeded into a 6-well plate and cultured for 10 days. Cells were fixed with $100 \%$ methanol and stained with $0.1 \%$ crystal violet for $30 \mathrm{~min}$. The number of the colonies was counted.

Western blotting. Total proteins were extracted from cells using a radioimmunoprecipitation lysis buffer (Thermo Fisher Scientific, Inc.) and protein concentrations were determined using a BCA Protein Assay kit (Beyotime Institute of Biotechnology). Protein samples (30 $\mu \mathrm{g} /$ lane) were fractionated using sodium dodecyl sulfate-polyacrylamide gel electrophoresis (SDS-PAGE) (10\% gels), transferred to polyvinylidene fluoride (PVDF) membranes (EMD Millipore,
Billerica, MA, USA), and blocked for $1 \mathrm{~h}$ with 5\% skimmed milk. Membranes were then incubated at room temperature overnight with the following primary antibodies: HMGB3 (1:500; cat. no. AF5507; R\&D Systems, Inc., Minneapolis, MN, USA), matrix metalloproteinase (MMP)7 (1:500; cat. no. sc-80205; Santa Cruz Biotechnology, Inc.), $\beta$-catenin (1:500; cat. no. 9562S; Cell Signaling Technology, MA, USA), c-MYC (1:500; cat. no. sc-373712; Santa Cruz Biotechnology, Inc.) and GAPDH (1:1,000; cat. no. AF0006; Beyotime Institute of Biotechnology). GAPDH served as a control. Subsequently, the membranes were incubated with horseradish peroxidase-conjugated goat anti-rabbit secondary antibodies (1:2,000; cat. no. sc-2004; Santa Cruz Biotechnology, Inc.) at room temperature for $1 \mathrm{~h}$. Protein expression levels were detected with enhanced chemiluminescence (ECL) detection solution (Beyotime Institute of Biotechnology).

Quantitative real-time reverse transcription-PCR ( $q P C R)$. The expression of miR-758 and 4 genes (HMGB3, $\beta$-catenin, MMP7 and c-MYC) was analyzed with SYBR-Green II (Takara Biotechnology Co., Ltd., Dalian, China) and a RT-qPCR system (MJ Research; Bio-Rad Laboratories, Inc., Hercules, CA, USA). RNA was isolated from cells and tissues using TRIzol (Invitrogen; Thermo Fisher Scientific, Inc.) following the manufacturer's protocol. The cDNA was synthesized using the PrimeScript ${ }^{\mathrm{TM}}$ RT Reagent Kit (Takara Biotechnology Co., Ltd.). Reverse transcription was performed on a GeneAmp PCR System 9700 (Applied Biosystems; Thermo Fisher Scientific, Inc.), and qPCR was performed on an ABI 7500 Real-Time PCR System (Applied Biosystems; Thermo Fisher Scientific, Inc.). All samples were processed at the same time to avoid inter-experiment variance. The thermocycling conditions were as follows: A holding step at $95^{\circ} \mathrm{C}$ for $30 \mathrm{sec}$, and 40 cycles at $95^{\circ} \mathrm{C}$ for $5 \mathrm{sec}$ and $60^{\circ} \mathrm{C}$ for $30 \mathrm{sec}$. The relative mRNA expression of miR-758 was analyzed as the inverse log of $\Delta \Delta \mathrm{Cq}$ and normalized to the reference gene U6 (10). The relative mRNA expression of HMGB3, MMP7, $\beta$-catenin, c-MYC was analyzed as the inverse $\log$ of $\Delta \Delta \mathrm{Cq}$ and normalized to the reference gene GAPDH (10). The primers were designed as follows: miR-758 forward, 5'-ACACTCCAGCTGGGA ACGATG3' and reverse, 5'-CTCAACTGGTGTCGTGGA GTCGGCA3'; U6 forward, 5'-TGCGGGTGCTCGCTTCGC AGC-3', and reverse, 5'-CCAGTGCAGGGTCCGAGGT-3'; HMGB3 forward, 5'-CAGCTTGATACCTGTGAATGGG-3' and reverse, 5'-TATCTGTGGTCGTGTGGGACT-3'; MMP7 forward, 5'-GTCTCTGGACG GCAGCTATG-3 and reverse, 5'-GATAGTCCTGAGCCTGTTCCC-3'; $\beta$-catenin forward, 5'-ACCTCCCAAGTCCTGTAT-3' and reverse, 5'-CCTGGT CCTCGTCATTTA-3'; c-MYC forward, 5'-CACAGCAAA CCTCCTCACAG-3' and reverse, 5'-GGATAGTCCTTCCGA GTGGA-3'; and GAPDH forward, 5'-CGGAGTCAACGG ATTTGGTCGTAT-3' and reverse, 5'-AGCCTTCTCCATGGT GGTGAAGAC-3'.

Transwell assay. For cell migration assays, $1 \times 10^{5}$ transfected cells in serum-free medium were added into the upper separate compartment of a Transwell chamber (Corning Incorporated, Corning, NY, USA). Medium containing 10\% FBS that was placed into the bottom chamber was used as a chemoattractant. For cell invasion assays, transfected cells were seeded 
into the upper chamber of the Transwell after adding diluted Matrigel. After $24 \mathrm{~h}$ of incubation for migration assays and $48 \mathrm{~h}$ of incubation for invasion assays at $37^{\circ} \mathrm{C}$ of a $5 \% \mathrm{CO}_{2}$ atmosphere, cells on the top surface of the filters that did not pass through the pores were removed from the upper chamber using a cotton swab, while cells on the bottom surface of the membrane that migrated or invaded through the pores were fixed with methanol and stained with $0.1 \%$ crystal violet for 30 min. Images from 5 different fields were captured and counted under a light microscope.

Bioinformatic prediction. To investigate the possible target gene of miR-758, the online prediction system, TargetScan (http://www.targetscan.org), was applied. TargetScan target gene prediction software identified the 2784-2791 site at the 3'-end of the 3'-UTR of HMGB3 mRNA as a possible site of action of miR-758.

Dual-luciferase reporter assay. Wild-type (Wt) and mutated (Mut) putative miR-758-binding sites in the HMGB3 3'-UTR region were cloned into the downstream region of the luciferase gene in the psiCHECK-2 ${ }^{\mathrm{TM}}$ Vector (Promega Corporation, Madison, WI, USA). For the reporter assay, 293T cells were co-transfected with Wt or mut HMGB3-3'-UTR vectors and miR-758 mimics or inhibitor. Luciferase activities were assessed with a Dual-Luciferase Reporter Assay Kit (Promega Corporation) following manufacturer's protocols. Data were normalized against the activity of the Renilla luciferase gene.

Statistical analysis. Statistical analyses were performed using SPSS version 16.0 (SPSS, Inc., Chicago, IL, USA). All experiments were performed in triplicate. Unless otherwise indicated, the data were evaluated as the mean \pm standard deviation. Differences between 2 groups were assessed using two-tailed Student's t-test. Data of $>2$ groups were assessed using one-way analysis of variance with post hoc Tukey's test. The correlations between miR-758 expression levels and the mRNA expression of HMGB3 levels in CC tissues were analyzed using Spearman's rank test. $\mathrm{P}<0.05$ was considered to indicate a statistically significant difference.

\section{Results}

The expression of miR-758 is downregulated in clinical samples and CC cells. The expression of miR-758 in tumor tissues and paired adjacent normal tissues isolated from $20 \mathrm{CC}$ patients was detected via qPCR. The results indicated that the expression of miR-758 was significantly reduced in CC tissues compared to adjacent normal tissues (Fig. 1A). Furthermore, we monitored miR-758 levels in CC cell lines (HeLa, C33A, $\mathrm{CaSki}$ and $\mathrm{SiHa}$ ) and a normal human cervical epithelial cell line (Ect1/E6E7). Results of qPCR revealed that the expression level of miR-758 was significantly decreased in CC cells compared to that of Ect1/E6E7 cells (Fig. 1B). These data illustrated that miR-758 may serve as a tumor suppressive gene in CC. The highest expression levels of miR-758 were detected in the $\mathrm{C} 33 \mathrm{~A}$ cells and the lowest expression was detected in the HeLa cells. Then, these two cell lines were selected for the further study. To discover the biological function of miR-758 in the progression of CC cells, gain- or loss-of-function assays
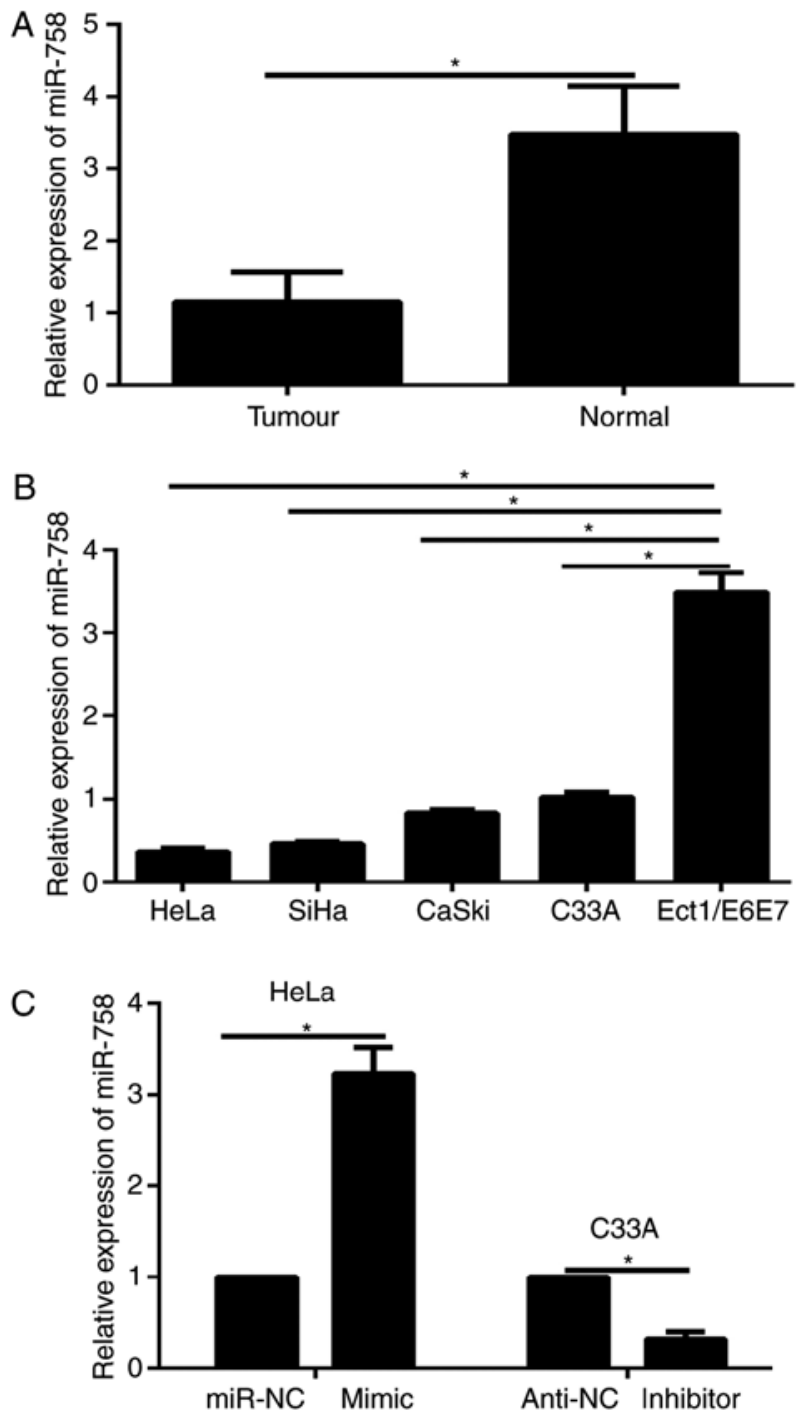

Figure 1. Expression of miR-758 is downregulated in clinical samples and CC cells. (A) The expression of miR-758 in tumor tissues and paired adjacent normal tissues was detected by qPCR. (B) The expression of miR-758 in $\mathrm{CC}$ cell lines (HeLa, C33A, CaSki and $\mathrm{SiHa}$ ) and a normal human cervical epithelial cell line (Ect1/E6E7) was detected by qPCR. (C) The expression of miR-758 in HeLa and C33A cells was detected by qPCR. ${ }^{*} \mathrm{P}<0.05$. CC, cervical cancer; qPCR, quantitative real-time reverse transcription-PCR.

were carried out by transfection of the miR-758 mimic or inhibitor in HeLa or C33A cells, respectively. Results of qPCR revealed that transfection of the miR-758 mimic significantly increased miR-758 expression in HeLa cells, while the miR-758 inhibitor significantly decreased the expression of miR-758 in C33A cells (Fig. 1C).

$H M G B 3$ is a direct target gene of miR-758. Analysis with the predictive database TargetScan suggested that HMGB3 is a putative target of miR-758 (Fig. 2A). Previous research has revealed that HMGB3 is a central player associated with cellular metastasis in some types of cancer. To illustrate that HMGB3 was a direct target gene of miR-758, 293T cells were co-transfected with Wt or mut HMGB3-3'-UTR vectors and miR-758 mimics or inhibitor. The expression of miR-758 in 293 T cells was revealed using qPCR. The results revealed that transfection of the miR-758 mimic significantly increased 
A

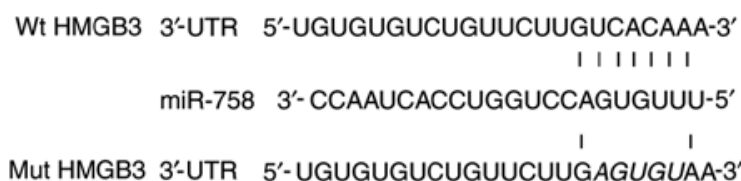

C

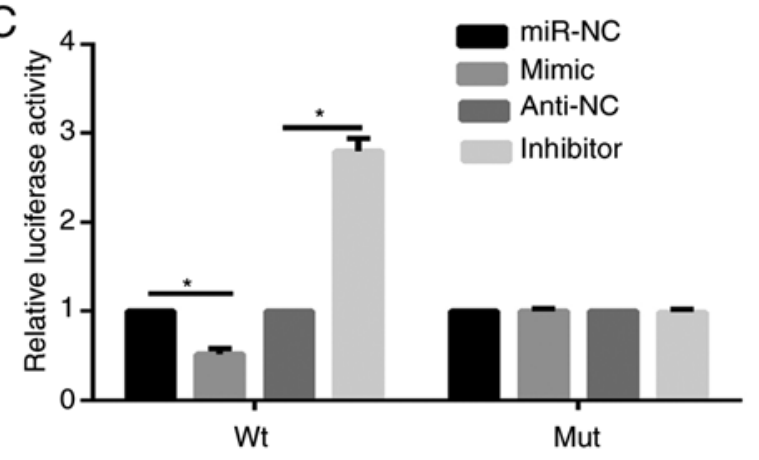

E

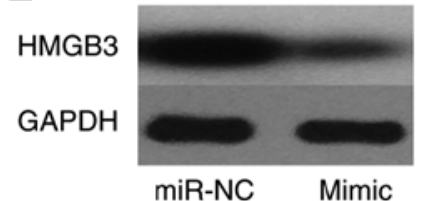

$F_{0}$

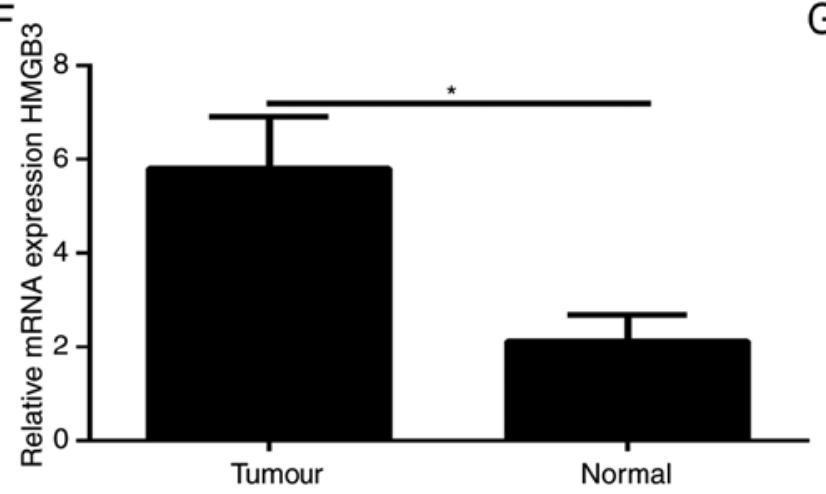

B

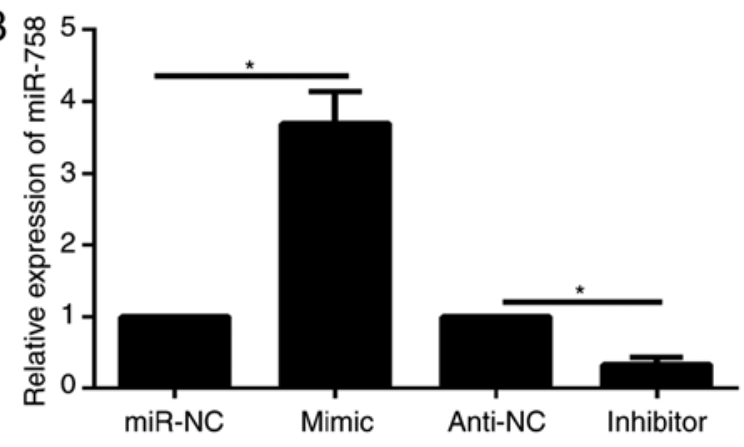

D

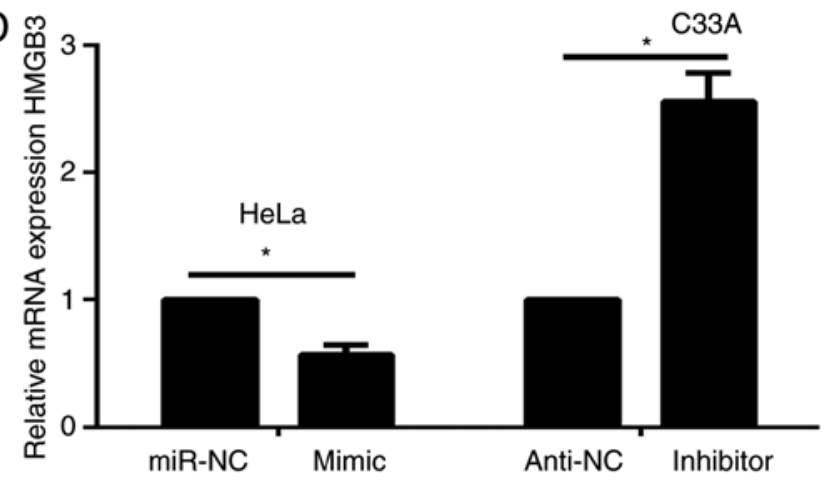

G

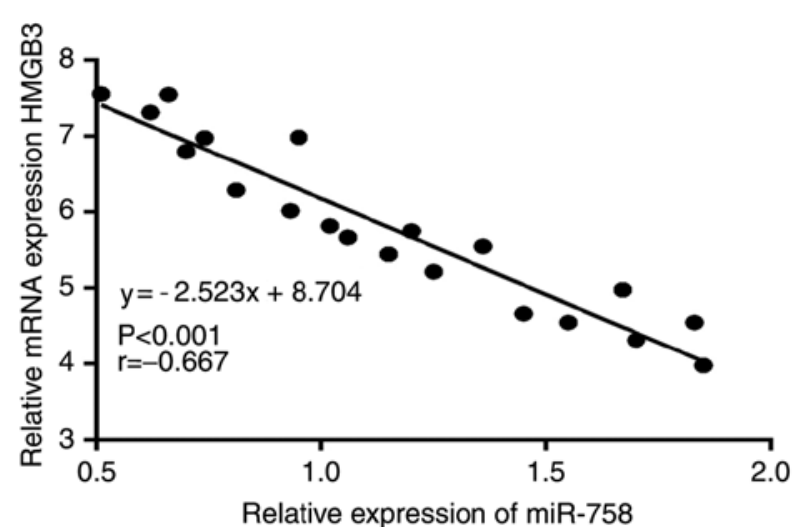

Figure 2. HMGB3 is a direct target gene of miR-758. (A) TargetScan prediction of miR-758 binding site on the 3'-UTR region of HMGB3. (B) The expression of miR-758 in 293T cells was detected by qPCR. (C) Luciferase reporter assay for HMGB3. (D) The mRNA expression of HMGB3 in HeLa and C33A cells was detected by qPCR. (E) Protein expression of HMGB3 in HeLa and C33A cells was detected by western blotting. (F) qPCR was used to detect the expression of HMGB3 in the CC tissues and corresponding adjacent normal tissues. (G) The expression of miR-758 and the mRNA expression of HMGB3 were negatively correlated in CC tissues. 'P<0.05. HMGB3, high mobility group box 3; 3'-UTR, 3'-untranslated region; qPCR, quantitative real-time reverse transcription-PCR; $\mathrm{CC}$, cervical cancer.

miR-758 expression in $293 \mathrm{~T}$ cells compared to the miR-NC group, while the miR-758 inhibitor markedly decreased the expression of miR-758 in $293 \mathrm{~T}$ cells compared to the anti-NC group (Fig. 2B). Luciferase reporter assays were carried out to explore whether miR-758 targets HMGB3 by binding to its 3'-UTR. Results of luciferase reporter assay demonstrated that the miR-758 mimic significantly decreased the luciferase activity of the wild-type 3'-UTR of HMGB3, while the miR-758 inhibitor significantly increased the luciferase activity of the wild-type HMGB3 3'-UTR (Fig. 2C). Furthermore, the results of qPCR and western blotting revealed that both the mRNA (Fig. 2D) and protein (Fig. 2E) levels of HMGB3 in the miR-758 mimic group were significantly decreased compared with the negative control group. However, the mRNA (Fig. 2D) and protein (Fig. 2E) expression level of HMGB3 were significantly upregulated in the miR-758 inhibitor group. Furthermore, the mRNA expression levels of HMGB3 in CC tumor tissues and paired adjacent normal tissues was analyzed via $\mathrm{qPCR}$. The results revealed that the expression level of HMGB3 was significantly enhanced in CC tissues compared with adjacent normal tissues (Fig. 2F). In addition, Spearman's correlation analysis revealed that the expression levels of miR-758 were negatively correlated with HMGB3 mRNA in $\mathrm{CC}$ tissues (Fig. 2G). In summary, these data indicated that miR-758 directly targeted HMGB3 by binding to its 3'-UTR region in $\mathrm{CC}$ cells.

miR-758 regulates the WNT/ $\beta$-catenin signaling pathway via HMGB3. To illustrate the mechanism of miR-758 in CC progression, we focused on the correlation of miR-758 and 
A

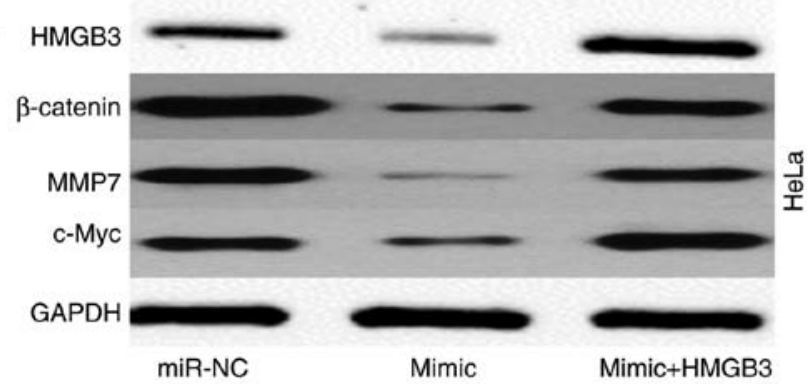

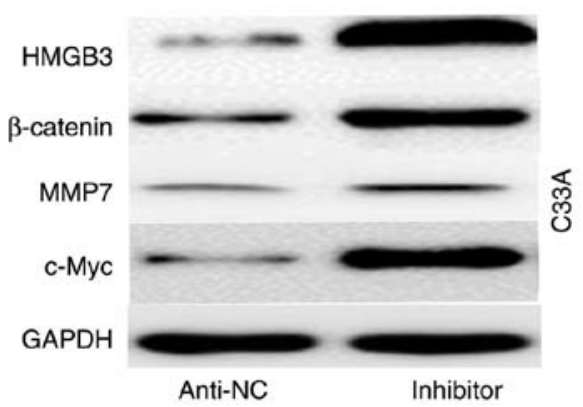

$\mathrm{B}$

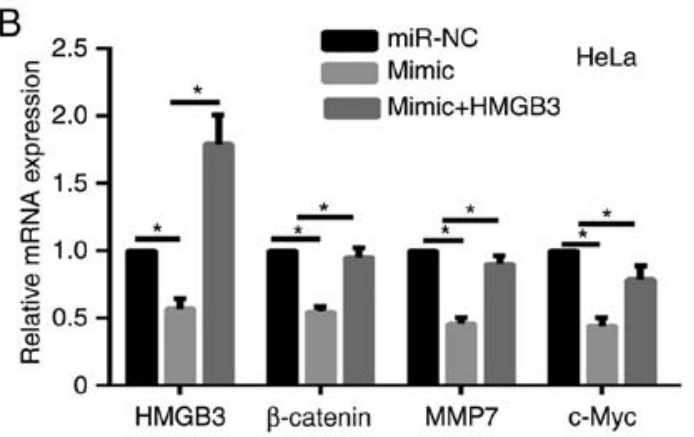

C

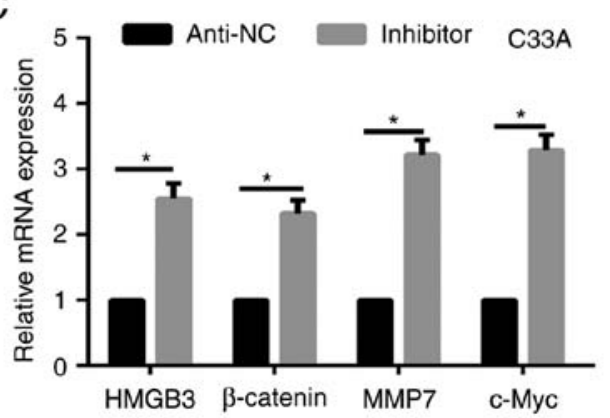

Figure 3. miR-758 regulates the WNT/ $\beta$-catenin signaling pathway via HMGB3. (A) Western blotting was carried out to detect HMGB3, $\beta$-catenin, MMP7 and c-Myc expression in HeLa and C33A cells. (B and C) qPCR was carried out to detect HMGB3, $\beta$-catenin, MMP7 and c-Myc mRNA expression in (B) HeLa and (C) C33A cells. ${ }^{*} \mathrm{P}<0.05$. HMGB3, high mobility group box 3; MMP, matrix metalloproteinase; qPCR, quantitative real-time reverse transcription-PCR.

WNT/ $\beta$-catenin signaling pathway. Results of western blotting and $\mathrm{qPCR}$ revealed that miR-758 mimic significantly decreased the expression of $\beta$-catenin and its target gene c-Myc, and MMP7 in HeLa cells (Fig. 3A and B), while miR-758 inhibitor markedly enhanced the expression of $\beta$-catenin, c-Myc, and MMP7 in C33A cells (Fig. 3A and C). A previous study revealed that $\mathrm{HMGB} 3$ negatively regulated the $\mathrm{WNT} / \beta$-catenin signaling pathway (11). Thus, whether miR-758 regulated the WNT/ $\beta$-catenin signaling pathway via HMGB3 was investigated. HeLa cells were co-transfected with miR-758 mimic and HMGB3 overexpression plasmid. The protein expression level of HMGB3 was detected by western blot assay (Fig. 3A). The results revealed that HMGB3 overexpression significantly enhanced the expression of $\beta$-catenin, c-Myc, and MMP7 decreased by the miR-758 mimic both at the protein and mRNA level (Fig. 3A and B). These data demonstrated that miR-758 regulated the WNT/ $\beta$-catenin signaling pathway via HMGB3.

miR-758 inhibits the proliferation of CC cells via HMGB3. To discover the biological function of miR-758 in the progression of CC cells, CCK-8 and colony formation assays were performed. Results of the CCK- 8 assay revealed that the viability of HeLa cells was significantly decreased by the miR-758 mimic (Fig. 4A), while opposite results were obtained in C33A cells transfected with the miR-758 inhibitor (Fig. 4B). Furthermore, the colony formation assay revealed that the transfection of the miR-758 mimic significantly decreased the proliferation of HeLa cells compared with the control group, while the miR-758 inhibitor significantly enhanced the proliferation of C33A cells (Fig. 4C). To ascertain whether HMGB3 could regulate the cell viability of miR-758 in CC cells, HeLa cells were co-transfected with miR-758 mimic and HMGB3 overexpression plasmid. The results of the CCK- 8 assay
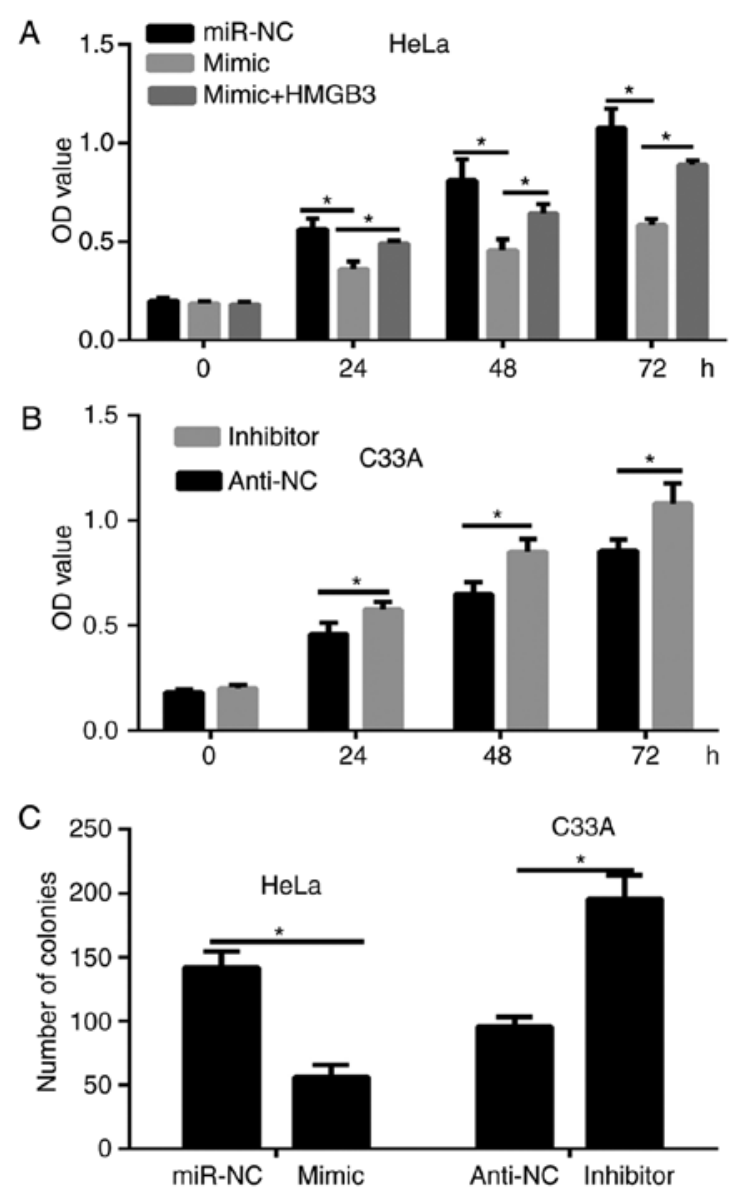

Figure 4. miR-758 mimic inhibits the viability of CC cells, while HMGB3 overexpression rescues the inhibitory effect induced by the miR-758 mimic. (A and B) CCK-8 assay was used to detect the cell viability of (A) HeLa and (B) C33A cells. (C) A colony formation assay was used to analyze the proliferation of $\mathrm{HeLa}$ and $\mathrm{C} 33 \mathrm{~A}$ cells. ${ }^{*} \mathrm{P}<0.05$. OD, optical density; $\mathrm{CC}$, cervical cancer; HMGB3, high mobility group box 3; CCK-8, Cell Counting Kit-8. 
A

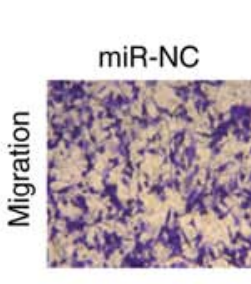

HeLa
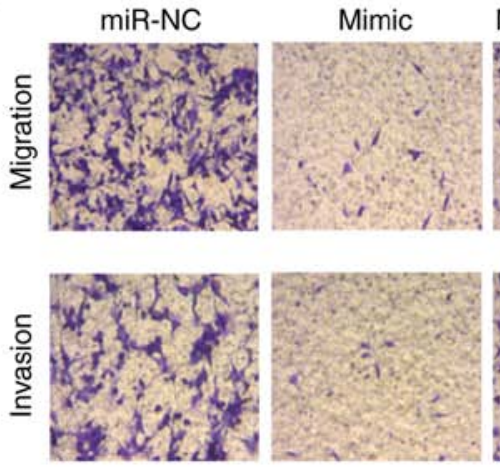
Mimic+HMGB3

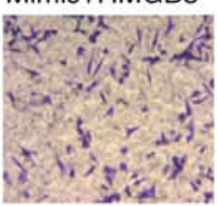

B
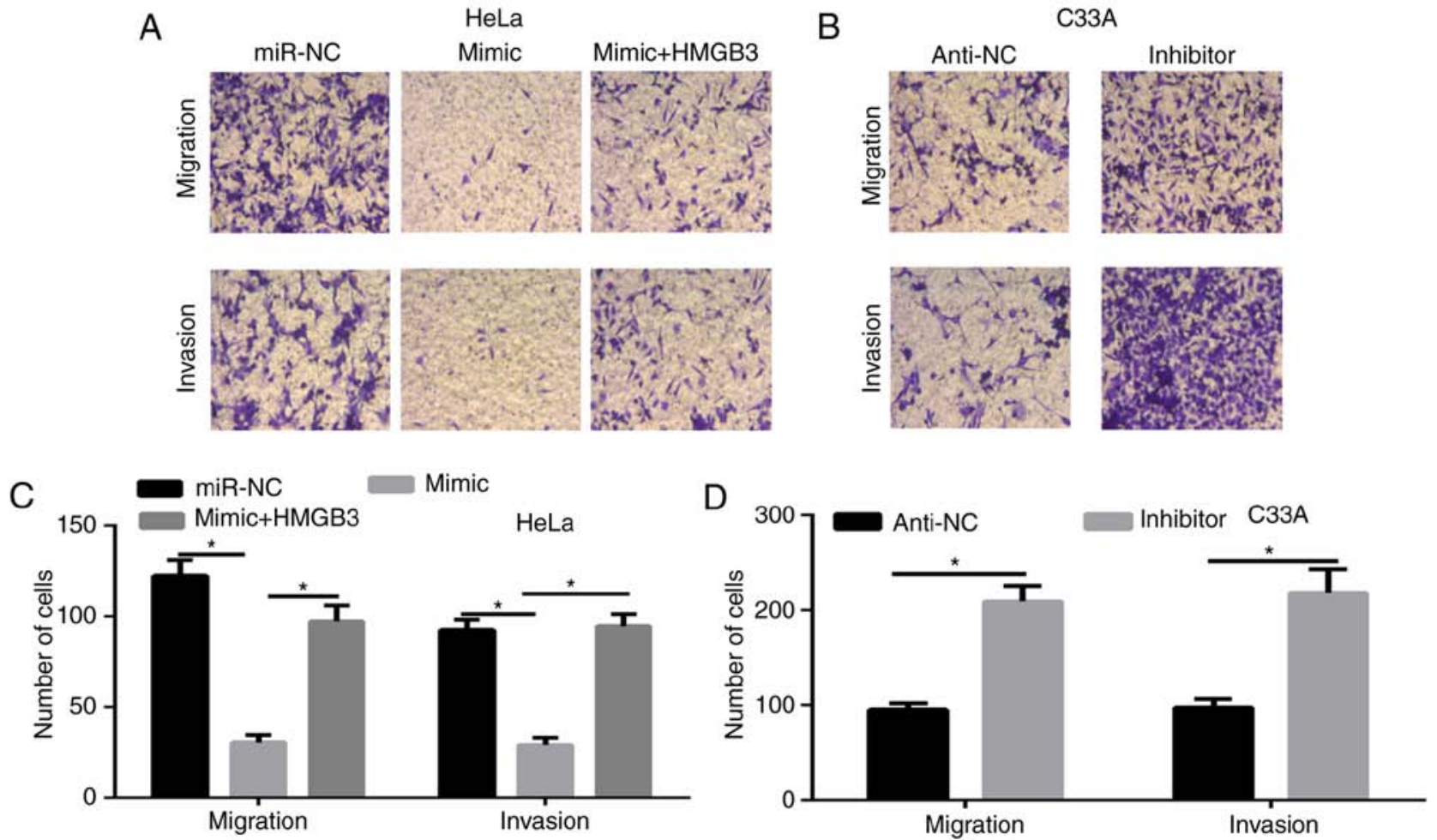

Figure 5. miR-758 inhibits migration and invasion in CC cells, while HMGB3 overexpression rescues the inhibitory effect induced by the miR-758 mimic. Transwell assays were used to detect the migration and invasion capabilities of CC cells. (A and B) Migratory and invasive cells were fixed, stained and counted and images were captured (magnification, $\mathrm{x} 100)$. ( C and D) Quantification of migration and invasion in HeLa and $\mathrm{C} 33 \mathrm{~A}$ cells in the lower chamber. ${ }^{*} \mathrm{P}<0.05$. CC, cervical cancer; HMGB3, high mobility group box 3.

(Fig. 4A) demonstrated that HMGB3 overexpression partially rescued the inhibitory effects of the miR-758 mimic on the viability of HeLa cells.

miR-758 inhibits the migration and invasion of CC cells. Cell migration and invasion are critical events in tumor progression (12). Thus, Transwell assays were employed to assess the function of the miR-758 on the migration and invasion of $\mathrm{CC}$ cells. The results revealed that the overexpression of miR-758 significantly decreased cell migration and invasion in $\mathrm{HeLa}$ cells, in contrast, the miR-758 inhibitor significantly enhanced cell migration and invasion in C33A cells (Fig. 5A-D). To ascertain whether HMGB3 regulated the cell migration and invasion of miR-758 in CC cells, HeLa cells were co-transfected with miR-758 mimic and HMGB3 overexpression plasmid. The results of the Transwell assays (Fig. 5A and C) demonstrated that HMGB3 overexpression partially rescued the inhibitory effects of miR-758 mimic on the migration and invasion of HeLa cells.

\section{Discussion}

Aberrant miR-758 expression has been discovered in glioblastoma, hepatocellular carcinoma and non-small lung cancer; furthermore, miR-758 has been revealed to inhibit the proliferation, migration and invasion of these cancer cells (6-8). miR-758 expression has been revealed to be significantly decreased in CC (9). However, the function and molecular mechanism of miR-758 in CC progression have not been well elucidated. Therefore, the molecular mechanism of miR-758 in the progression of CC must be further revealed.
In the present study, we demonstrated that miR-758 was downregulated in CC tissues and cell lines, and it may serve as a novel tumor suppressor in CC. miR-758 upregulation significantly suppressed tumor growth, migration and invasion, whereas miR-758 silencing enhanced tumor progression. In the mechanism study, we illustrated that miR-758 acted as a tumor suppressor by negatively regulating the WNT/ $\beta$-catenin signaling pathway by directly targeting HMGB3.

miR-758 serves as a tumor suppressor by regulating different targets in various types of cancers. In the progression of glioblastoma, miR-758 inhibited tumor progression by directly targeting BTB domain-containing protein 20 (6). In hepatocellular carcinoma, miR-758 served as a tumor suppressor and played a crucial role in inhibiting proliferation, migration and invasion by targeting MDM2 and mTOR (8). In the CC tissues, miR-758 may regulate the infiltration and invasion of $\mathrm{CC}$ by targeting matrix extracellular phosphoglycoprotein (9). However, miR-758 regulates the expression of multiple genes. Therefore, the molecular mechanism of miR-758 in the inhibitory function of CC progression was investigated. In the present study, the correlation of miR-758 and HMGB3 was demonstrated. HMGB3 belongs to the high-mobility group box family, which includes HMGB1, HMGB2, HMGB3 and HMGB4 (13). The high-mobility group box family plays an important role in the progression of several types of cancer $(11,14-17)$. HMGB3 has been regulated by some different miRNAs in several types of cancer. However, the correlation of miRNA and HMGB3 has not been illustrated in CC. Therefore, this correlation was determined. In the present study, it was revealed that HMGB3 expression was significantly decreased after downregulation of 
miR-758 in CC cells, but enhanced by the miR-785 inhibitor. Moreover, miR-758 expression was negatively correlated with HMGB3 mRNA expression in CC cell tissues. Finally, it was demonstrated that HMGB3 overexpression rescued the inhibitory function role of the miR-758 mimic. In summary, our results demonstrated that HMGB3 is a molecular and functional target of miR-758. The WNT/ $\beta$-catenin signaling pathway has been revealed to promote cancer progression in some types of cancer, including CC (18). HMGB3 can regulate the WNT/ $\beta$-catenin signaling pathway in colorectal cancer (11). In the present study, it was demonstrated that miR-758 negatively regulated the WNT/ $\beta$-catenin signaling pathway. In the rescue experiments, it was demonstrated that HMGB3 overexpression enhanced the expression of $\beta$-catenin and its target genes MMP7 and c-Myc. These data demonstrated that miR-758 promoted $\mathrm{CC}$ growth, migration and invasion by negatively regulating the $\mathrm{WNT} / \beta$-catenin signaling pathway by directly targeting HMGB3.

In summary, the present study demonstrated that miR-758 was downregulated in CC tissues and cell lines. miR-758 also functioned as a tumor suppressor of $\mathrm{CC}$ growth by targeting HMGB3. This newly identified miR-758/HMGB3 link provides new insight into the mechanisms underlying $\mathrm{CC}$ development and suggested that targeting the miR-758/HMGB3 axis may represent a promising therapeutic strategy for $\mathrm{CC}$ treatment. However, further studies are required to determine the exact mechanism of decreased miR-758 expression during the progression of $\mathrm{CC}$ and to further explore other possible molecular mechanisms of miR-758 in CC.

\section{Acknowledgements}

Not applicable.

\section{Funding}

No funding was received.

\section{Availability of data and materials}

The datasets used during the present study are available from the corresponding author upon reasonable request.

\section{Authors' contributions}

TS and XHH conceived and designed the experiments. TS, $\mathrm{XHH}$ and BL conducted all of the experiments. XHH and BL wrote and revised the manuscript. All authors read and approved the final manuscript.

\section{Ethics approval and consent to participate}

The present study involving human samples was approved by the Medical Ethics Committee of Weifang Maternity and Child Care Hospital (Weifang, China) and written informed consent was obtained from all patients.

\section{Patient consent for publication}

\section{Competing interests}

The authors declare that they have no competing interests.

\section{References}

1. Siegel R, Ma J, Zou Z and Jemal A: Cancer statistics, 2014. CA Cancer J Clin 64: 9-29, 2014.

2. Smith RA, Brooks D, Cokkinides V, Saslow D and Brawley OW: Cancer screening in the United States, 2013: A review of current American cancer society guidelines, current issues in cancer screening, and new guidance on cervical cancer screening and lung cancer screening. CA Cancer J Clin 63: 88-105, 2013.

3. Dai S, Lu Y, Long Y, Lai Y, Du P, Ding N and Yao D: Prognostic value of microRNAs in cervical carcinoma: A systematic review and meta-analysis. Oncotarget 7: 35369-35378, 2016.

4. Kanekura K, Nishi H, Isaka K and Kuroda M: MicroRNA and gynecologic cancers. J Obstet Gynaecol Res 42: 612-617, 2016.

5. Kwan JY, Psarianos P, Bruce JP, Yip KW and Liu FF: The complexity of microRNAs in human cancer. J Radiat Res 57 (Suppl 1): i106-i111, 2016.

6. Liu J, Jiang J, Hui X, Wang W, Fang D and Ding L: Mir-758-5p suppresses glioblastoma proliferation, migration and invasion by targeting ZBTB20. Cell Physiol Biochem 48: 2074-2083, 2018.

7. Wang $\mathrm{S}$ and Jiang $\mathrm{M}$ : The long non-coding RNA-DANCR exerts oncogenic functions in non-small cell lung cancer via miR-758-3p. Biomed Pharmacother 103: 94-100, 2018.

8. Jiang D, Cho W, Li Z, Xu X, Qu Y, Jiang Z, Guo L and Xu G: MiR-758-3p suppresses proliferation, migration and invasion of hepatocellular carcinoma cells via targeting MDM2 and mTOR. Biomed Pharmacother 96: 535-544, 2017.

9. Meng X, Zhao Y, Wang J, Gao Z, Geng Q and Liu X: Regulatory roles of miRNA-758 and matrix extracellular phosphoglycoprotein in cervical cancer. Exp Ther Med 14: 2789-2794, 2017.

10. Livak KJ and Schmittgen TD: Analysis of relative gene expression data using real-time quantitative PCR and the 2(-Delta Delta C(T)) method. Methods 25: 402-408, 2001.

11. Zhang Z, Chang Y, Zhang J, Lu Y, Zheng L, Hu Y, Zhang F, Li X, Zhang $\mathrm{W}$ and $\mathrm{Li} \mathrm{X}$ : HMGB3 promotes growth and migration in colorectal cancer by regulating WNT/ $\beta$-catenin pathway. PLoS One 12: e0179741, 2017.

12. Vu M, Yu J, Awolude OA and Chuang L: Cervical cancer worldwide. Curr Probl Cancer 42: 457-465, 2018.

13. Nemeth MJ, Curtis DJ, Kirby MR, Garrett-Beal LJ, Seidel NE, Cline AP and Bodine DM: Hmgb3: An HMG-box family member expressed in primitive hematopoietic cells that inhibits myeloid and B-cell differentiation. Blood 102: 1298-1306, 2003.

14. Chen X and Zeng L: Ginkgo biloba extract 761 enhances 5-fluorouracil chemosensitivity in colorectal cancer cells through regulation of high mobility group-box 3 expression. Am J Transl Res 10: 1773-1783, 2018.

15. Yamada Y, Nishikawa R, Kato M, Okato A, Arai T, Kojima S, Yamazaki K, Naya Y, Ichikawa T and Seki N: Regulation of HMGB3 by antitumor miR-205-5p inhibits cancer cell aggressiveness and is involved in prostate cancer pathogenesis. J Hum Genet 63: 195-205, 2018.

16. Gao J, Zou Z, Gao J, Zhang H, Lin Z, Zhang Y, Luo X, Liu C, Xie J and Cai C: Increased expression of HMGB3: A novel independent prognostic marker of worse outcome in patients with esophageal squamous cell carcinoma. Int J Clin Exp Pathol 8: 345-352, 2015.

17. Song N, Liu B, Wu JL, Zhang RF, Duan L, He WS and Zhang CM: Prognostic value of HMGB3 expression in patients with non-small cell lung cancer. Tumour Biol 34: 2599-2603, 2013.

18. Bahrami A, Hasanzadeh M, ShahidSales S, Yousefi Z, Kadkhodayan S, Farazestanian M, Joudi Mashhad M, Gharib M, Mahdi Hassanian S and Avan A: Clinical significance and prognosis value of Wnt signaling pathway in cervical cancer. J Cell Biochem 118: 3028-3033, 2017.

This work is licensed under a Creative Commons Attribution-NonCommercial-NoDerivatives 4.0 International (CC BY-NC-ND 4.0) License. 